\title{
A multidisciplinary approach for a patient with neck pain \& dizziness following a motor vehicle accident:
}

\section{a case report}

\begin{abstract}
Following a motor vehicle accident, there is a cascade of mechanical, chemical, and psychological events that can occur. ${ }^{1,2}$ Many times this arduous process of assess-referassess can be detrimental to the physical and mental rehabilitation following this traumatic event. Efficient, streamlined processes in place may reduce the overall healthcare burden following a motor vehicle accident (MVA). We present a case study of a 56-year-old male with complaints of vertigo, neck pain, and disequilibrium. Following emergency department clearance with imaging techniques and referral for further evaluation. The patient was seen five weeks following his injury at The American Institute of Balance (AIB). The AIB is a multidisciplinary clinic with specialists in vestibular Audiology and Physiotherapy with utilization of comprehensive neurodiagnostic and assessment protocols. A confirmed right ear, posterior canal Benign Paroxysmal Positional Vertigo(BPPV-PC) was successfully treated with the Gans Repositioning Maneuver (GRM). This was followed by physiotherapy interventions for his complaints of neck pain and imbalance, post clearance of the BPPVPC. He was seen in the clinic for a total of three weeks, with a progression of home exercise given each week. At the end of his physiotherapy, he was discharged with no further complaints of vertigo and significantly improved mobility, strength, and subjective reports for his neck pain. This case study demonstrates the necessity for proper evaluation and comprehensive treatment when multiple comorbidities follow traumatic events. This ensures time-efficient management and successful and measurable treatment outcomes.
\end{abstract}

Volume 5 Issue 3 - 2020

\author{
Steven E Spinks, Joseph T Sakumura, Richard \\ E Gans
}

The American Institute of Balance, USA

\begin{abstract}
Correspondence: Steven E Spinks, PT, DPT, OCS, COMT, FAAOMPT,Vice President of Research, The American Institute of Balance, USA, Tel 7273985728, Fax 72739849|4,
\end{abstract}

Emails.spiks@dizzy.com

Received: May 30, 2020 | Published: June 12, 2020

Keywords: dizziness, neck pain, benign paroxysmal positional vertigo, canalith repositioning, cervicogenic dizziness

Abbreviations: MVA, motor vehicle accident; BPPV-PC, benign paroxysmal positional vertigo- posterior canal; GRM, gans repositioning maneuver; WAD, whiplash associated disorder; VOR, vestibular-ocular reflex; VNG, videonystagmography

\section{Introduction}

Motor vehicle accidents account for nearly $10 \%$ of all emergency department visits per year in the United States. ${ }^{3}$ According to the Centers for Disease Control, neck and back pain consists of $24 \%$ of these post-accident visits. ${ }^{3}$ Of those patients reporting neck pain following an MVA, approximately $50 \%$ report concomitant dizziness. ${ }^{8,33}$ Typically, these patients are managed in the acute phase with bracing, ${ }^{4,5}$ pharmacological intervention, ${ }^{6,7}$ and referral to ${ }^{8}$ in the event of no red flags. Dizziness alone, represents about $4 \%$ of all emergency department visits. ${ }^{9}$ Persistence of symptoms may extend past the acute phase and transition into the chronic phase where we know yellow flags ${ }^{10-12}$ may begin to show, including fear avoidance patterns, anxiety, stress, and depression. This is the transition from what was originally termed as whiplash to whiplash associated disorders. ${ }^{13}$ One component of whiplash associated disorder (WAD) that is often underdiagnosed and treated is dizziness.

While there is a plethora of research on the topic of orthopedic assessment for patients with neck pain and whiplash associated disorders, ${ }^{14,15}$ there is little research on the dizziness that follows. A comprehensive evaluation is often needed for patients following an MVA, including orthopedic, psychology, and rehabilitative assessment from physiotherapy and occupational therapy. Assessment for dizziness is often done by the physiotherapist in the clinic, where many may only specialize in balance rehabilitation without a focus on the vestibular system or access to neurodiagnostic testing (e.g. kinetic rotary chair, computerized dynamic posturography). Comprehensive neurodiagnostic assessment can assist in the rehabilitation treatment diagnosis ${ }^{16}$ for efficacious treatments, especially as third-party payors begin to transition for pay-for-performance models.

\section{Case description}

\section{History}

An active, and generally healthy, 56-year-old male reports to the AIB multidisciplinary clinic following a rear-end collision, motor vehicle accident that occurred five weeks ago. He reports wearing a seat belt with no airbag deployment. Emergency Medical Services were called to the scene, where the patient recalls fogginess and significant posterior neck pain. Per their protocol, the patient was taken to the Emergency Department for further assessment. Computerized Tomography (CT) was performed and revealed moderate degenerative changes along C4-C6. Cervical spondylosis is often asymptomatic and there is little evidence to support a correlative factor between abnormal imaging and reports of pain. ${ }^{17-19}$ Magnetic Resonance Imaging (MRI) revealed minimal broad-based posterior disc herniation at the level of C3-C4. Following assessment, for which he was cleared, he was seen by his primary care physician the next day. He describes minimal neck pain through his life with no significant events that he can recall. He reports localized posterior neck pain along the right side, but does not travel distally, and positional vertigo, which he describes as "everything is spinning around me". 


\section{Examination}

A detailed and thorough examination was performed both by a vestibular Audiologist and Physiotherapist at The American Institute of Balance, a specialized multidisciplinary clinic in Tampa, Florida, USA. Subjective examination included subjective questionnaires, past medical history, and current complaints. Of note, the subjective questionnaires included the Dizziness Handicap Inventory, Neck Disability Index, and the Tampa Scale of Kinesiophobia. The Dizziness Handicap Inventory is a 25 -item, well validated selfreported questionnaire ${ }^{20}$ that has a 5 -item subscale that is predictive of Benign Paroxysmal Positional Vertigo (BPPV). The Neck Disability Index is a 10-item questionnaire on functional capabilities related to the cervical spine that has high validity and reliability ${ }^{8,9}$ with excellent test-rest reliability and internal consistency. ${ }^{22}$ Due to the high fear avoidance patterns noted in many patients following an accident with chronic neck pain, ${ }^{23,24}$ the Tampa Scale of Kinesiophobia was administered to determine limitations to rehabilitation potential due to fear of movement. This questionnaire has been shown to be a valid and reliable psychometric measure. ${ }^{25,26}$ Subjective questioning regarding symptoms mechanics were assessed with the "SPINS" model for subjective assessment of dizziness. This assessed the severity of the patient's symptoms, persistence, irritability, nature (pathophysiological or causative factors), and stability of their presentation. The patient reported worsening symptoms over the past two weeks, most noted when lying down and rolling over in bed to turn off his alarm in the morning. Moderate neck pain was present during most of his transitional movements and improved in the middle of the day, then returned to moderate pain in the evening prior to going to bed. The patient's primary physical complaints consisted of neck pain, difficulty rotating and extending their neck due to pain and fear, and reports of poor balance when walking his dog at night. A complete neurodiagnostic workup was performed by a vestibular Audiologist and a physical and functional evaluation was performed by a vestibular physiotherapist. Red flag screening was performed by the physiotherapist prior to cervical assessment for vertebrobasilar artery insufficiency and upper cervical instability tests, all of which were negative. Table 1 summarizes the examination findings from this patient.

Table I Abbreviations \& key

\begin{tabular}{|c|c|c|c|c|c|c|c|}
\hline & & Initial Evaluation & & & Discharge & & \\
\hline \multirow[t]{5}{*}{ Subjective } & NPRS & $6 / 10$ & & & $1 / 10$ & & \\
\hline & Dizziness Rating & $5 / 10$ & & & $0 / 10$ & & \\
\hline & $\mathrm{DHI}$ & $48 \%$ & & & $2 \%$ & & \\
\hline & NDI & $27 / 50$ & & & $2 / 50$ & & \\
\hline & TSK & $47 / 68$ & & & $19 / 68$ & & \\
\hline \multicolumn{8}{|l|}{ Objective } \\
\hline \multirow[t]{8}{*}{ Physiotherapy } & Cervical AROM ${ }^{2}$ & Left & Neutral & Right & Left & Neutral & Right \\
\hline & Flexion & & 36 & & & 64 & \\
\hline & Extension & & 19 & & & 59 & \\
\hline & Lateral Flexion & 8 & & 17 & 18 & & 20 \\
\hline & Rotation & 49 & & 38 & 82 & & 84 \\
\hline & SPNT & + & & + & - & & - \\
\hline & Cervical Proprioception & \multicolumn{3}{|l|}{$1 \mathrm{lcm}$ from neutral } & \multicolumn{3}{|c|}{$1.5 \mathrm{~cm}$ from neutral } \\
\hline & Craniocervical Flexion Test & \multicolumn{3}{|l|}{18 seconds, 7/10 NPRS } & \multicolumn{3}{|c|}{43 seconds, I/I0 NPRS } \\
\hline \multirow[t]{7}{*}{ Neuro diagnostic } & CDP & + & & + & - & & - \\
\hline & Kinetic Rotary Chair & - & & - & - & & - \\
\hline & Gaze & - & & - & - & & - \\
\hline & Headshake & - & & - & - & & - \\
\hline & Side-Lying & - & & + & - & & - \\
\hline & Hallpike & & & & & & \\
\hline & Positionals & - & & - & - & & - \\
\hline
\end{tabular}

Abbreviations: NPRS, Numeric pain rating scale; DHI, dizziness handicap inventory; NDI, neck disability index;TSK, tampa scale of kinesiophobia; SPNT, smooth pursuit neck torsion test; CDP, computerized dynamic posturography

Key: \&, measurements taken via long arm goniometer and in degrees 


\section{Neurodiagnostic examination}

Postural stability and the vestibulo-spinal reflex (VSR) were evaluated with computerized dynamic posturography (CDP). The pattern of sway deviation and falls on vision denied and dynamic surface conditions were suggestive of vestibular involvement. This pattern is frequently seen in patients with benign paroxysmal positional vertigo (BPPV) and typically normalizes after successful canalith repositioning. Following successful treatment and amelioration of the signs and symptoms of BPPV, testing was repeated and found to be within age and height normalized parameters. Figure 1 for results and explanation of findings for the CDP.
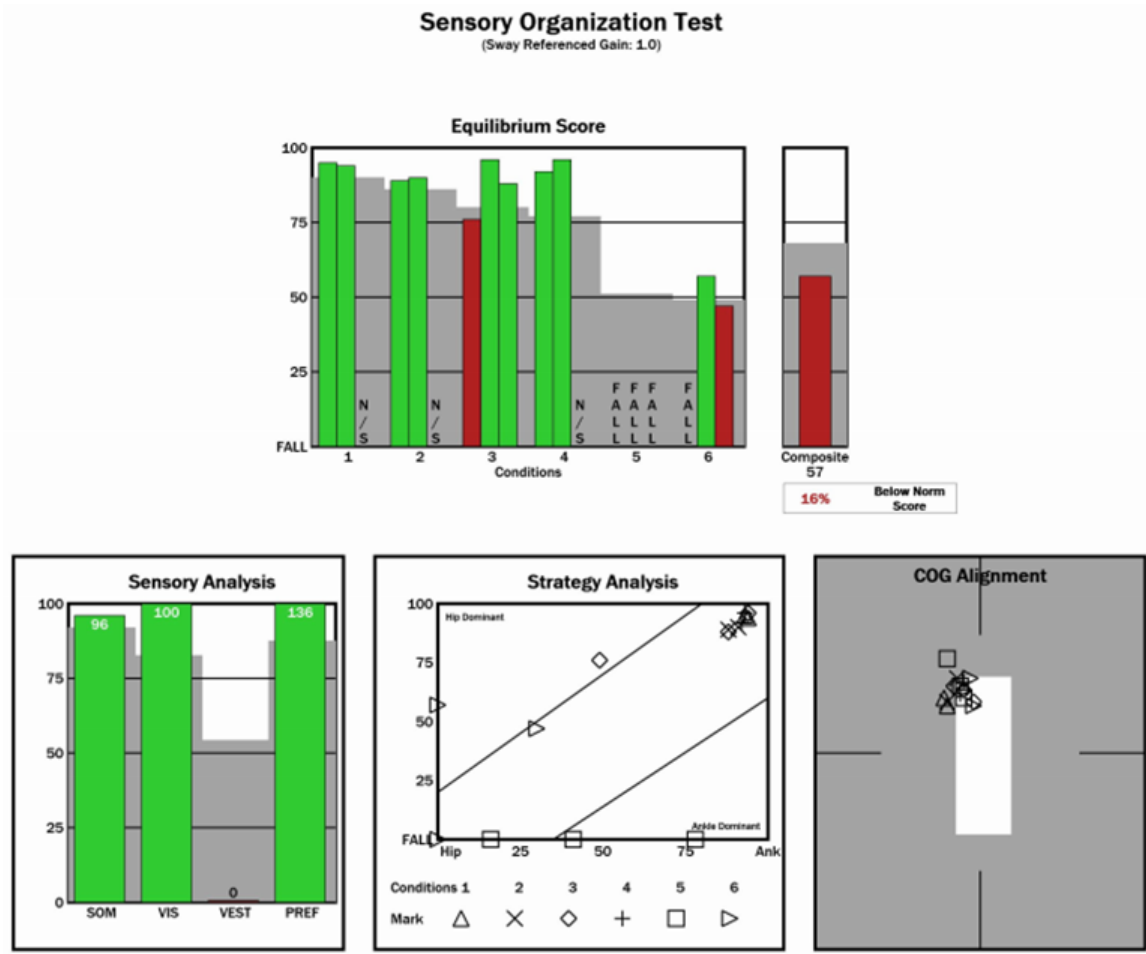

Figure IA Test results at initial evaluation (pre-treatment), indicated abnormal findings on computerized dynamic posturography(CDP) with normal step velocity function on rotary chair.

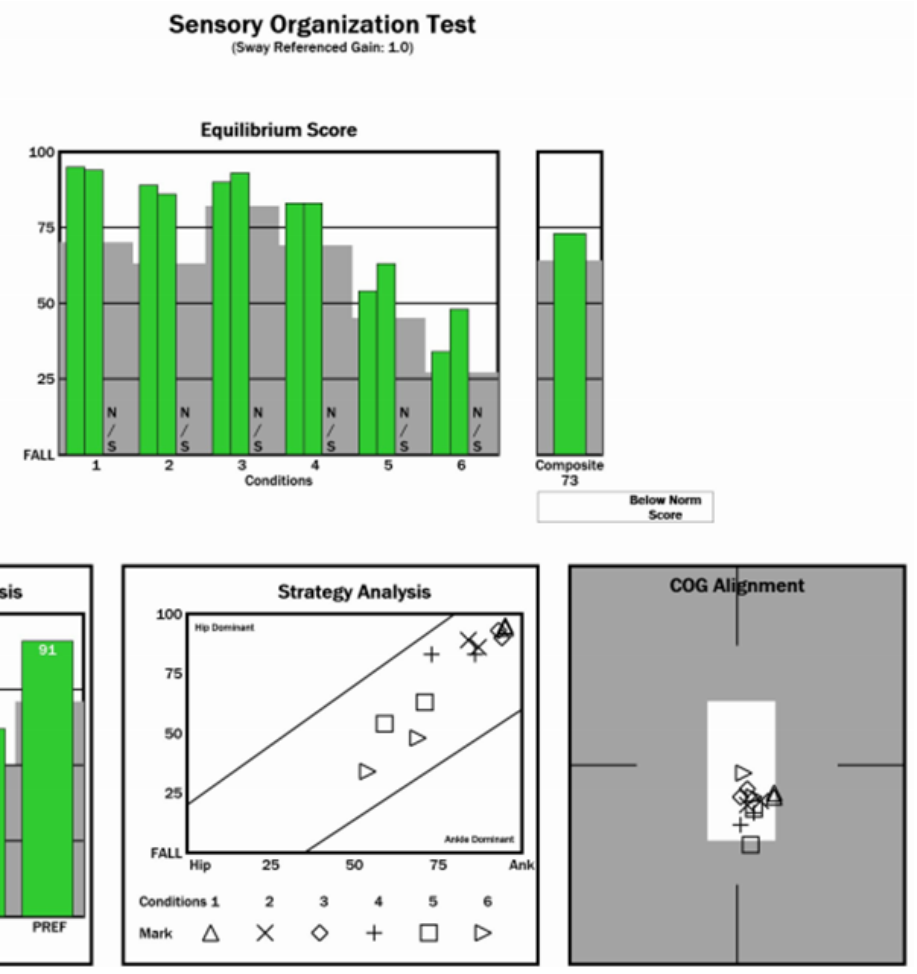

Figure IB Post BPPV treatment recovery of postural stability function on CDP. 
Vestibulo-ocular reflex (VOR) was assessed via Videonystagmography (VNG) and kinetic rotary chair evaluation. VNG testing was negative for any spontaneous, gaze evoked, or posthead shaking nystagmus. Side-lying Hallpike testing was performed due to the patient's cervical comorbidities and revealed a right posterior canal benign paroxysmal positional vertigo (BPPV) which was evidenced by rotary torsional nystagmus and accompanying vertigo that fatigued after approximately 15 seconds. Positional testing was otherwise unremarkable for any clinically significant nystagmus. As can be seen in Figure 2, step velocity gain and phase on rotary chair testing was within normal limits with bidirectional rotation. This was consistent with intact peripheral and central vestibular function other than the active BPPV at the time of initial evaluation.

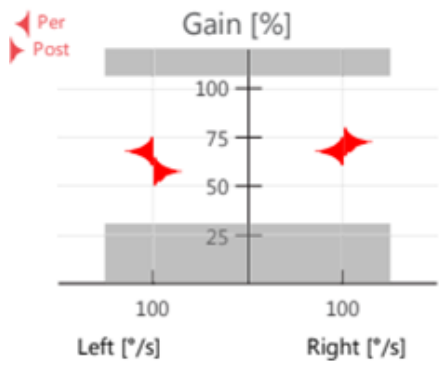

\section{Step Rotation}

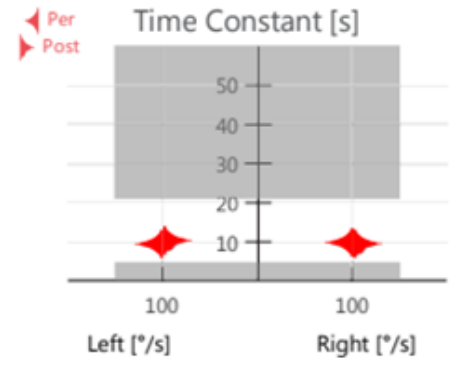

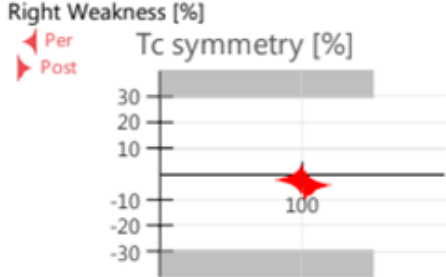

Left Weakness [\%]

Figure 2 Rotary chair assessment with step rotation was within normal limits for all parameters; gain, time constant, and symmetry.

\section{Intervention}

The multidisciplinary approach taken with this patient was based on an impairment-based intervention strategy that categorized treatments based on patient positioning for interventions and patient's symptoms. Due to the fact that many manual therapy interventions and exercises are done in supine, the patient's complaints of vertigo in this position would be addressed first. The assessment and treatment of the patient's right posterior canal BPPV was done via the Gans Repositioning Maneuver in a fully supported manner. This maneuver has been shown to be safe and efficacious for patients with cervical spine pathology, most notably those with reduced cervical extension and rotation. ${ }^{27,28}$ This maneuver is highly efficacious with an average $90 \%$ success rate after just 1.25 treatments. ${ }^{29}$ BPPV clearance was achieved after 2 intra-session treatments. This was evidenced by retest of the right posterior canal with negative findings. As per the therapists' protocols, prophylactic treatment was performed after these negative findings. Assessment of the horizontal canals following treatment was also negative for any otolith involvement.
Following amelioration of vertiginous symptoms via successful repositioning maneuvers, the patient underwent treatment for his posterior neck pain. Based upon the patient's impairments noted in his subjective and objective examination, it was determined that a combination of manual therapy and exercise would benefit him the most. The literature is supportive of this rationale for a multimodal approach $^{29-31}$ to build patient rapport and independence in everyday activities while not creating a dependency-model. This consisted of pain neuroscience education with the importance of movement, resilience of the spine, and for resolution of symptoms that can be managed independently. He demonstrated good understanding following the first visit's education. His next follow up visit was two days after and consisted of mid-cervical rotational mobilization, deep neck flexor activation exercises, and upper extremity aerobic activities. He was subsequently seen for two more weeks with a reduction in overall manual therapy interventions and progressed into independence of home exercise programs for cervical strengthening and upper extremity functional movements. Table 2 shows the matched impairment-interventions that were chosen for this patient.

Table 2 Abbreviations \& Key

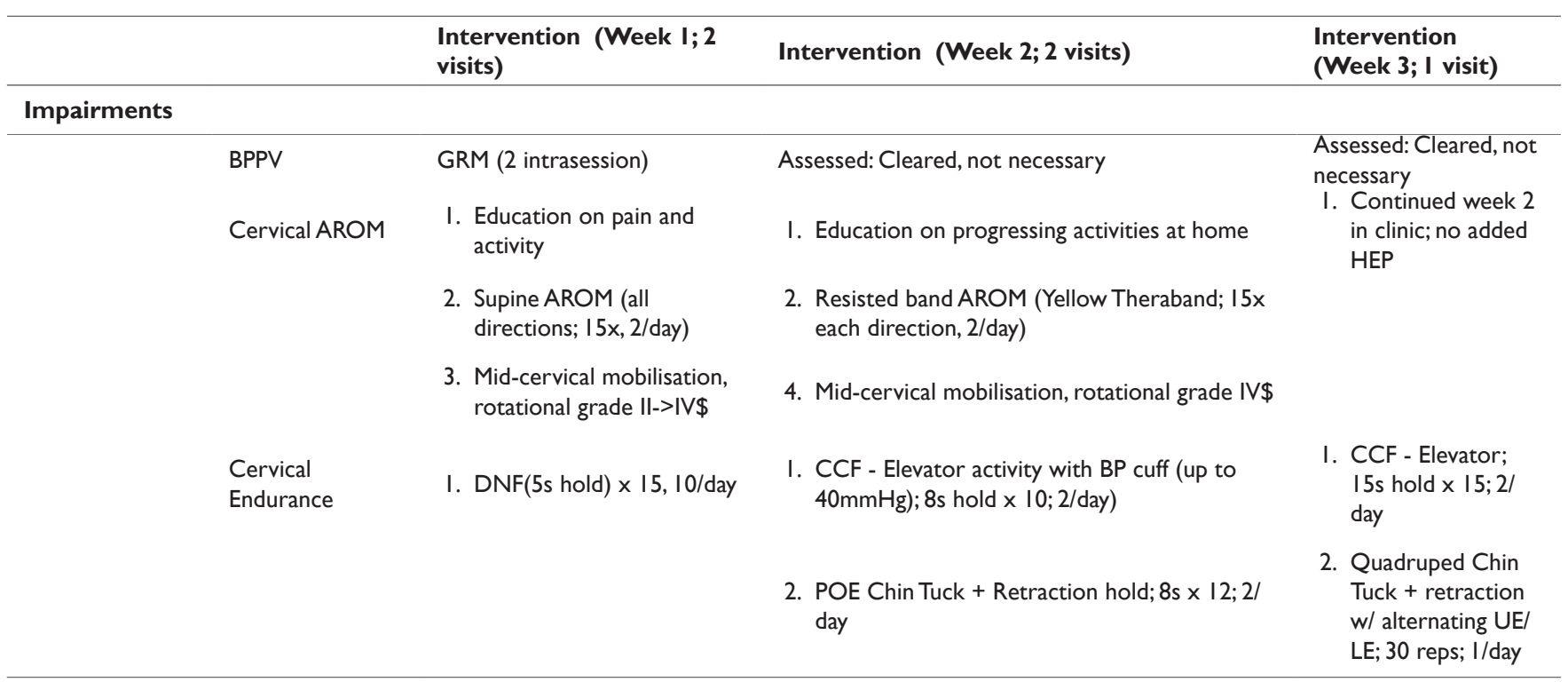


Table Continued...

\begin{tabular}{|c|c|c|c|c|}
\hline & & $\begin{array}{l}\text { Intervention (Week I; } 2 \\
\text { visits) }\end{array}$ & Intervention (Week 2; 2 visits) & $\begin{array}{l}\text { Intervention } \\
\text { (Week 3; I visit) }\end{array}$ \\
\hline \multicolumn{5}{|l|}{ Impairments } \\
\hline & $\begin{array}{l}\text { Cervical } \\
\text { Proprioception }\end{array}$ & Did not prescribe & $\begin{array}{l}\text { I. Laser-mounted hat; straight lines all directions } \\
\text { on wall; } 30 \text { s each direction; } 2 / \text { day seated }\end{array}$ & $\begin{array}{l}\text { I. Continued } \\
\text { laser-mounted } \\
\text { positioning; } \\
\text { standing with } \\
\text { varying surface; } \\
30 \text { s each direction; } \\
2 / \text { day }\end{array}$ \\
\hline & Postural Stability & $\begin{array}{l}\text { I. EO, Narrow BOS, } 30 \text { s x } \\
\text { I0; } 2 \text { /day }\end{array}$ & I. EC,Tandem (B), I5s x 10;2/day & $\begin{array}{l}\text { I. EO, slight head } \\
\text { shakes with } \\
\text { ambulation; } 6 \mathrm{~m}\end{array}$ \\
\hline & & $\begin{array}{l}\text { 2. EC, Narrow BOS, } 10 \text { s } x \\
\text { I0; } 2 / \text { day }\end{array}$ & $\begin{array}{l}\text { 2. EC, Narrow BOS, slow head shakes } \times 10 \text { s; } 2 / \\
\text { day }\end{array}$ & \\
\hline
\end{tabular}

Abbreviations: BPPV, benign paroxysmal positional vertigo; GRM, gans repositioning maneuver; AROM, active range of motion; HEP, home exercise program; DNF, deep neck flexor; CCF, craniocervical flexion; BP, blood pressure; POE, prone on elbows; UE/LE, upper extremity, lower extremity; EO, eyes open; BOS, base of support; EC, eyes closed; (B), bilateral

Key: \$, Maitland-Australian physiotherapy grade of mobilisation

\section{Discussion}

There is minimal research demonstrating the correlation between neck pain and dizziness in the multidisciplinary setting. The current available literature is most focused on either physiotherapy intervention with bedside evaluation techniques ${ }^{32-36}$ or neurodiagnostics alone with no interventions paired. ${ }^{37,38}$ This patient received a structured, multidisciplinary evaluation and treatment for his complaints of dizziness and neck pain following a motor vehicle accident. While radiographic imaging was performed, demonstrating arthritic changes and disc involvement, his symptoms did not necessarily correlate with these pathological changes. However, based on the accident, fear avoidance patterns, and clinical decision making, a hybrid approach to managing his BPPV was taken utilizing the Gans Repositioning Maneuver where he was treated successfully in one visit.

This success allowed for proper physiotherapy intervention in manual therapy, exercise, and most importantly - education. Proper triage, via neurodiagnostic testing, allowed the physiotherapist to target his interventions at the patient's impairments for efficient management of the patient's complaints and, ultimately, return to his activities in a pain-free and dizzy-free environment

\section{Conclusion}

This case described the diagnosis and management of a patient with concomitant vertigo and cervicogenic dizziness following a motor vehicle accident. Factors that assisted in the clinical decisionmaking model were (1) neurodiagnostic testing for inner ear pathologies, (2) communication between the vestibular Audiologist and Physiotherapist, and (3) incorporation of evidence-based treatment for the patient's BPPV and cervicogenic dizziness with progression to safely return to his everyday activities.

\section{Funding}

None.

\section{Acknowledgments}

None.

\section{Conflicts of interest}

The authors declare no conflicts of interest.

\section{References}

1. Kim MB, Chang SW, Lim GC, et al. Clinical characteristics of patients with dizziness after motor vehicle accident. $J$ Int Adv Otol. 2017;13(3):374-378.

2. Rabe S, Zoellner T, Beauducel A, et al. Changes in brain electrical activity after cognitive behavioral therapy for posttraumatic stress disorder in patients injured in motor vehicle accidents. Psychosom Med. 2008;70(1):13-19.

3. https://www.cdc.gov/nchs/products/databriefs/db185.html

4. Endo K, Ichimaru K, Komagata M, et al. Cervical vertigo and dizziness after whiplash injury. Eur Spine J. 2006;15(6):886-890.

5. Treleaven J, Peterson G, Ludvigsson ML, et al. Balance, dizziness and proprioception in patients with chronic whiplash associated disorders complaining of dizziness: A prospective randomized study comparing three exercise programs. Man Ther. 2016;22:122-130.

6. Storaci R, Manelli A, Schiavone N, et al. Whiplash injury and oculomotor dysfunctions: clinical-posturographic correlations. Eur Spine J. 2006;15(12):1811-1816.

7. Whitcroft KL, Massouh L, Amirfeyz R, et al. A comparison of neck movement in the soft cervical collar and rigid cervical brace in healthy subjects. J Manipulative PhysiolTher. 2011;34(2):119-122.

8. Cleland JA, Childs JD, Whitman JM. Psychometric properties of the neck disability index and numeric pain rating scale in patients with mechanical neck pain. Arch Phys Med Rehabil. 2008;89(1):69-74.

9. Jull G, Kenardy J, Hendrikz J, et al. Management of acute whiplash: a randomized controlled trial of multidisciplinary stratified treatments. Pain. 2013;154(9):1798-1806.

10. Lemming D, Sörensen J, Graven-Nielsen T, et al. Managing chronic whiplash associated pain with a combination of low-dose opioid (remifentanil) and NMDA-antagonist (ketamine). Eur $J$ Pain. 2007;11(7):719-732.

11. Poorbaugh K, Brismée J-M, Phelps V, et al. Late whiplash syndrome: A clinical science approach to evidence-based diagnosis and management. Pain Pract. 2008;8(1):65-89. 
12. Chua K, Gans RE, Spinks S. Demographic and clinical characteristics of BPPV patients a retrospective large cohort study of patients. Journal of Otolaryngology-ENT Research. 2020;12(12).

13. Bussières AE, Stewart G, Al-Zoubi F, et al. The treatment of neck painassociated disorders and whiplash-associated disorders: a clinical practice guideline. J Manipulative PhysiolTher. 2016;39(8):523-564.e27.

14. Ms HT, Johnston V, Treleaven JM, et al. The Neck pain driving index (NPDI) for chronic whiplash-associated disorders: development, reliability, and validity assessment. Spine J. 12(10):912-920.

15. Oka H, Matsudaira K, Fujii T, et al. Risk factors for prolonged treatment of whiplash-associated disorders. PLoS One. 2015;10(7):e0132191.

16. https://www.dizzy.com/wp-content/uploads/ENTMJ20-Gans Rutherford-2.pdf

17. Brinjikji W, Luetmer PH, Comstock B, et al. Systematic literature review of imaging features of spinal degeneration in asymptomatic populations. AJNR Am J Neuroradiol. 2015;36(4):811-816.

18. Coronado R, Hudson B, Sheets C, et al. Correlation of magnetic resonance imaging findings and reported symptoms in patients with chronic cervical dysfunction. J Man ManipTher. 2009;17(3):148-153.

19. Rudy IS, Poulos A, Owen L, et al. The correlation of radiographic findings and patient symptomatology in cervical degenerative joint disease: a cross-sectional study. Chiropr Man Therap. 2015;23:9.

20. Whitney SL, Marchetti GF, Morris LO. Usefulness of the dizziness handicap inventory in the screening for benign paroxysmal positional vertigo. OtolNeurotol. 2005;26(5):1027-1033.

21. Sterling M. A proposed new classification system for whiplash associated disorders--implications for assessment and management. Man Ther. 2004;9(2):60-70.

22. McCarthy MJH, Grevitt MP, Silcocks P, et al. The reliability of the Vernon and Mior neck disability index, and its validity compared with the short form-36 health survey questionnaire. Eur Spine J. 2007;16(12):21112117.

23. Buitenhuis J, de Jong PJ. Fear avoidance and illness beliefs in posttraumatic neck pain. Spine . 2011;36(25 Suppl):S238-S243.

24. Nederhand MJ, Ijzerman MJ, Hermens HJ, et al. Predictive value of fear avoidance in developing chronic neck pain disability: consequences for clinical decision making. Arch Phys Med Rehabil. 2004;85(3):496-501.

25. Neblett R, Hartzell MM, Mayer TG, et al. Establishing clinically meaningful severity levels for the Tampa Scale for Kinesiophobia (TSK13). Eur J Pain. 2016;20(5):701-710.

26. Woby SR, Roach NK, Urmston M, et al. Psychometric properties of the TSK-11: a shortened version of the Tampa Scale for Kinesiophobia. Pain. 2005;117(1-2):137-144.
27. Gans RE, Harrington-Gans PA. Treatment efficacy of benign paroxysmal positional vertigo (bppv) with canalith repositioning maneuver and Semontliberatory maneuver in 376 patients. In: Seminars in Hearing. Vol 23. Copyright $(2002$ by Thieme Medical Publishers, Inc., 333 Seventh Avenue, New; 2002:129-142.

28. Omara A, Mosaad DM, Mohamed AS, et al. Epley repositioning maneuver versus Gans repositioning maneuver on postural instability in elderly patients with benign paroxysmal positional vertigo. The Egyptian Journal of Otolaryngology. 2017;33(2):518.

29. Roberts RA, Gans RE, Montaudo RL. Efficacy of a new treatment maneuver for posterior canal benign paroxysmal positional vertigo. $J \mathrm{Am}$ AcadAudiol. 2006;17(8):598-604.

30. Reid SA, Callister R, Katekar MG, et al. Effects of cervical spine manual therapy on range of motion, head repositioning, and balance in participants with cervicogenic dizziness: a randomized controlled trial. Arch Phys Med Rehabil. 2014;95(9):1603-1612.

31. Vincent K, Maigne J-Y, Fischhoff C, et al. Systematic review of manual therapies for nonspecific neck pain. Joint Bone Spine. 2013;80(5):508515.

32. Huijbregts P, Vidal P. Dizziness in orthopaedic physical therapy practice: classification and pathophysiology. J Man ManipTher. 2004;12(4):199214

33. Amer Al-Saif, Physical therapy examination for patients with cervicogenic dizziness. J Nov Physiother. 2013;3(3):1-4.

34. Hall CD, Herdman SJ, Whitney SL, et al. Vestibular Rehabilitation for Peripheral Vestibular Hypofunction: An Evidence-Based Clinical Practice Guideline: from the american physical therapy association neurology section. J Neurol Phys Ther. 2016;40(2):124-155.

35. L'Heureux-Lebeau B, Godbout A, et al. Evaluation of paraclinical tests in the diagnosis of cervicogenic dizziness. Otol Neurotol. 2014;35(10):18581865 .

36. Polaczkiewicz L, Olszewski J. Analyze causes and results of VNG examinations in patients with vertigo and balance disorders in the private ENT practice. Otolaryngol Pol. 2019;74(2):23-30.

37. Kamper SJ, Rebbeck TJ, Maher CG, et al. Course and prognostic factors of whiplash: a systematic review and meta-analysis. Pain. 2008;138(3):617629.

38. Walton DM, Macdermid JC, GiorgianniAA, et al. Risk factors for persistent problems following acute whiplash injury: update of a systematic review and meta-analysis. J Orthop Sports Phys Ther. 2013;43(2):31-43. 